\title{
STATISTICAL ANALYSIS OF PERIODICITY OF JOVIAN S-BURST
}

\author{
A. Kumamoto*, S. Kakimoto ${ }^{\dagger}$, Y. Sasaki ${ }^{\ddagger}$, H. Misawa*, Y. Katoh*, \\ F. Tsuchiya*, and B. Cecconi ${ }^{\S}$
}

\begin{abstract}
Statistical analysis of the periodicity of Jovian S-bursts has been performed based on the dataset from high time resolution observations of Jovian decametric radiation since 1985 at observatories of Tohoku University. Jovian S-bursts consist of narrow-band emissions with a negative frequency drift rate. The repetition frequency of the emissions is 2-400 Hz [Carr and Reyes, 1999, JGR 104, A11, 25127-25142], which is suggested to be similar with the eigen-frequency of the Jovian Ionospheric Alfven resonator (IAR) [Ergun et al., 2006, JGR 111, A06212; Su et al., 2006, JGR 111, A06211]. The eigen-frequency of IAR depends on the Alfvén velocity and the scale height of the ionosphere [Lysak, 1991, JGR 96, 1553-1568]. Therefore, we can expect that the repetition frequency of Jovian S-burst emissions provides us information of the Jovian ionosphere around the Io footprint.

In the analysis we used spectrograms with a bandwidth of $2 \mathrm{MHz}$ selected from a frequency range from 20 to $40 \mathrm{MHz}$ and a time resolution of $2 \mathrm{~ms}$ (from 1985 to 1992), and all those with a frequency range from 20 to $40 \mathrm{MHz}$ and a time resolution of $0.8 \mathrm{~ms}$ (from 2012), and we derived the repetition frequency of Jovian S-burst emissions. The analysis shows that the repetition frequency of Jovian S-burst emissions becomes small as the solar zenith angle (SZA) at the Io footprint increases. The decrease of eigen-frequency of JIAR suggests that the scale height of the Jovian nightside ionosphere increases probably due to heating by auroral electrons. Because the size of the dataset of Jovian S-bursts with high time resolution obtained from Tohoku University observatories is too huge to provide via network, we provide the dataset to researchers on request. In addition, we are also preparing metadata archive for providing information such as operation time of high time resolution receiver at Tohoku University observatories in the system of Virtual Observatory (VO) [Erard et al., 2014, Astron. and Computing 7, 71-80; http://voparis-europlanet-dev.obspm.fr/] with support of JSPS Bilateral Program "Coordinated observational and theoretical researches for Jovian and Kronian auroral radio emissions" (http://c.gp.tohoku.ac.jp/sakura/). The progress of the development of the metadata archives of Jovian S-burst for VO will also be shown in the presentation.
\end{abstract}

\footnotetext{
* Tohoku University, Sendai, Japan

$\dagger$ TEPCO, Tokyo, Japan

$\ddagger$ Komaba High School, Nippon Institute of Technology, Japan

$\S$ LESIA, Observatoire de Paris, Meudon, France
} 
